\title{
Preparation of experimental and numerical research on unsteady cavitating flow around hydrofoil
}

\author{
Martin Komárek ${ }^{1, a}$, Milan Sedláŕ ${ }^{1}$, Michal Vyroubal $^{1}$, Patrik Zima $^{2}$, Miloš Müller $^{3}$ and Tomáš Pálka \\ ${ }^{1}$ Centre of Hydraulic Research, J. Sigmunda 190, 78349 Lutín, Czech Republic \\ ${ }^{2}$ Institute of Thermomechanics ASCR, v.v.i., Dolejškova 5, 18200 Praha 8, Czech Republic \\ ${ }^{3}$ Faculty of Mechanical Engineering, Technical University of Liberec, Hálkova 6, 46117 Liberec, Czech Republic \\ ${ }^{4}$ SIGMA Research and Development Institute, J. Sigmunda 79, 78350 Lutín, Czech Republic
}

\begin{abstract}
This paper describes the preparation of the experimental setup and the results of the CFD analysis for the investigation of the unsteady cavitating flow around a prismatic NACA 2412 hydrofoil at different flow conditions. The experimental research is carried out in the cavitation water tunnel located in the Centre of Hydraulic Research in Lutín. The tunnel is integrated into the closed horizontal loop of the hydraulic test rig. The transparent test section provides a full view of the flow around the hydrofoil. The hydrofoil incidence angle is fully adjustable. Two variants of hydrofoil of the same geometry have been designed. One variant is equipped with pressure tap holes for the measurement of the static pressure, and the other variant is covered with a grid of five PVDF films, fully integrated in the hydrofoil. The PVDF films enable to measure the impact forces exerted on the hydrofoil surface by the collapsing cavitation structures. The high-frequency pressure oscillations downstream of the hydrofoil trailing edge are measured using the PVDF hydrophone. The cavitating flow is visualized using two simultaneously operating high-speed cameras providing the top and side views of the flow. A preliminary CFD analysis using the ANSYS CFX package helped to determine the flow regimes for the experimental study. The obtained CFD results agree well with the preliminary measurements.
\end{abstract}

\section{Introduction}

Unsteady cavitation phenomena represent a serious problem for hydrodynamic machines operated at a wide range of flow rates. At off-design conditions cavitation instabilities can cause very high level of noise and dangerous vibrations. Moreover, coherent collapses of large unsteady vapour structures result in significant erosion of the material in hydraulic machines due to cavitation and can severely shorten machine useful life, disrupt machine operation and efficiency as a result of altered geometry, and even cause catastrophic structural failures leading to high maintenance costs. Bubble clouds, which grow and collapse concurrently, are known to be particularly destructive.

Cavitation instabilities inside hydrodynamic machines have been studied extensively especially for the case of inducers, which are primarily designed for the operation at high cavitation intensities. The research of cavitation instabilities is usually based on visualizations and the frequency analysis to determine the dominant frequencies and compare them with the rotational frequency as well as the fundamental frequency of the inducer blade passage excitation [1-3]. The typical frequencies of some cavitation instabilities in inducers can be found e.g. in [3]. Recently, several studies of cavitation instabilities in hydrodynamic machines have appeared [4-6]. These studies are based mainly on numerical simulations. In [6] it has been shown that the cavitation instability in the backflow vortices in front of the pump impeller appears in a limited interval of cavitation numbers. This is caused by the interaction of the separated flow with the cavitation regions. When the cavitation number is sufficiently high (the cavitation intensity is low), the cavities formed on the impeller blades are small and relatively stable. By contrast, when the cavitation number is very low, the cavitation regions can block the blade-toblade passages of the impeller and the backflow practically vanishes.

The interaction between the separated flow and cavitation results in periodic pressure oscillations with high amplitudes leading to significant forces acting on the blades. The background mechanisms of the interaction are very complicated. Experimental studies of the interaction inside a complex geometry of a real machine are highly expensive and time-consuming and in some cases practically impossible. Therefore, in order to study the interaction and the consequent cavitation instabilities, an experimental setup with a simplified geometry (prismatic NACA 2412 hydrofoil) has been prepared in the cavitation facility in the Centre of Hydraulic Research in Lutín. This experimental research is accompanied with

\footnotetext{
a Corresponding author: m.komarek@sigma.cz
} 
a comprehensive CFD analysis for a wide range of flow regimes. Advanced turbulence models including SASSST and Reynolds-stress models have been tested to capture the highly unsteady phenomena on the hydrofoil.

\section{Assembly of cavitation tunnel}

The cavitation tunnel in the Centre of Hydraulic Research was built in 2007 and was initially used to investigate erosion triggered by cavitation [7]. The tunnel was rebuilt in 2013 to enable to study highly unsteady cavitation phenomena. The facility is a horizontal plane water tunnel for isolated hydrofoils (Figure 1). It is formed by a closed loop equipped with compressor and vacuum pumps capable of creating different pressure levels while maintaining constant volume flow rate. The water reservoir has the capacity of $35 \mathrm{~m}^{3}$. In order to remove solid particles from the flowing medium a filtration unit was added in the bypass line between the end of the discharge piping and the reservoir. The filter removes particles with the minimum size of $10 \mu \mathrm{m}$.

The rectangular test section has inner dimensions $150 \times 150 \times 500 \mathrm{~mm}$ and is made of organic glass to enable visual access from all sides (Figure 2). The flow is driven by a variable-speed axial-flow pump and can attain the maximum velocity $25 \mathrm{~m} / \mathrm{s}$ at the inlet of the test section.

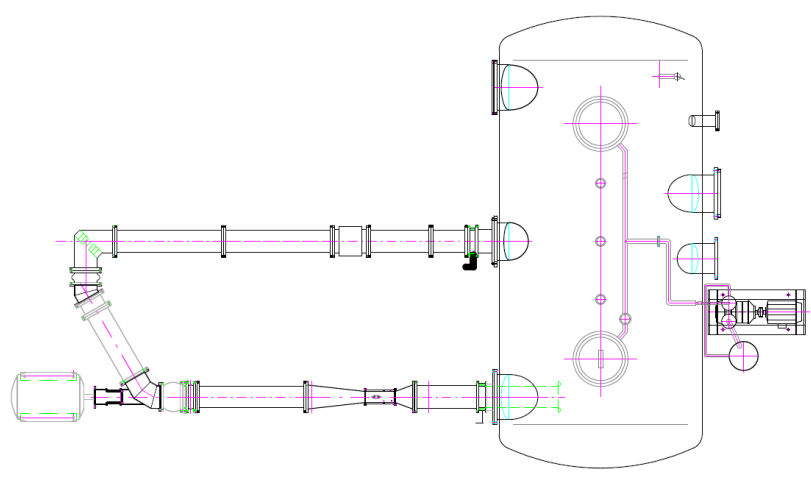

Figure 1. Horizontal plane test rig with the cavitation test section in the inner loop.

One of the requirements for the design of the tunnel was to enable parallel operation of both the experimental rig for performing standard hydraulic pump tests and the cavitation branch for research purposes, sharing the same reservoir and a number of supporting devices and utilities. Therefore, a special branch of the test rig has been designed, which enables to run permanent cavitation tests independently of the standard hydraulic test branch. The tunnel loop is made of DN300 stainless-steel pipes and is separated from the reservoir by two flap valves. Another requirement was to ensure a sufficiently uniform velocity at the inlet to the test section as the contraction area ratio of the tunnel overal convergent section decreased to 3.14 (compared to 8.7 in the previous design described in [7]). For this purpose, a special extension of the inlet piping was added, protruding into the reservoir (see Figure 3). For simplicity, the reservoir's inner soothing screens are not shown in Figure 3. The new configuration has allowed us to achieve the velocity uniformity at the inlet to the test section within $\pm 1 \%$ (excluding the boundary layer).

\section{Description of experimental hydrofoils}

The hydrofoils used for the cavitation tests are prismatic, with a chord length of $120 \mathrm{~mm}$, which corresponds to the span/chord ratio 1.25 . The span/chord ratio influences significantly the blockage effect of the tunnel wals. The current ratio is large enough to eliminate the importance of the boundary-layer flow on the vertical walls of the test section. The profile incidence angle can vary between $0^{\circ}$ and $\pm 180^{\circ}$. The NACA 2412 hydrofoil has been chosen as the test case for its similarity to the blade profiles used in hydrodynamic machines.

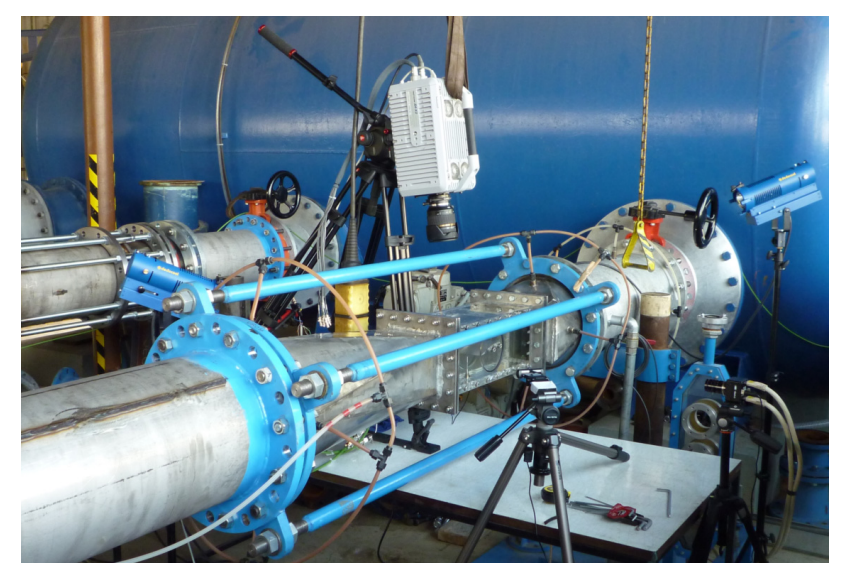

Figure 2. Cavitation tunnel.
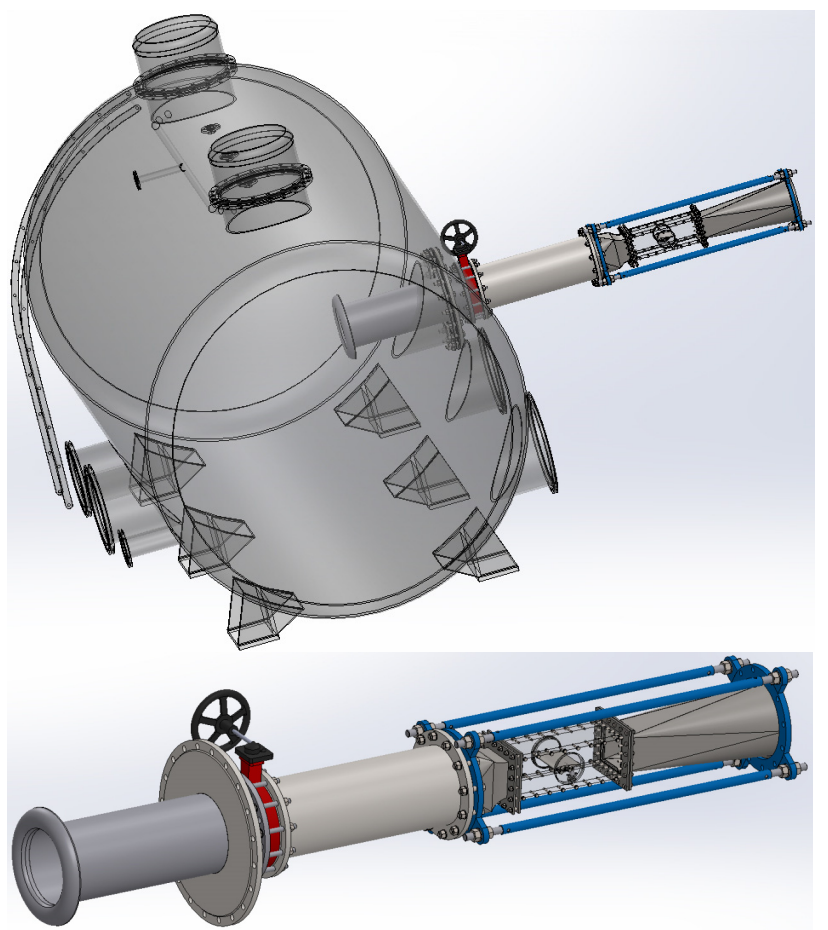

Figure 3. Suction extension of the inlet piping protruding into the water reservoir.

The main objective of the research conducted in the cavitation tunnel is to study the dependence of the unsteady cavitation phenomena on the cavitation intensity 
(expressed using the cavitation number) at relatively high incidence angles. Several incidence angles have been considered, however, the incidence angle of $8^{\circ}$ has been selected for the majority of the tests to enable comparison with the results published in [8]. However, there are some differences between this work and [8] as the authors of [8] used a different hydrofoil (Clark Y) and a lower span/chord ratio 0.815 .

Two variants of the NACA 2412 hydrofoil have been designed. The first variant has two tap holes for the measurement of the static pressure at midspan - one at the leading edge and one on the suction side at $40 \%$ of the chord length (Figure 4). The choice of only two measurement points was determined by the requirement to minimize the $\mathrm{V}$-shaped disturbance of the flow caused by the presence of the tap holes. The PVDF hydrophone is installed downstream of the trailing edge at $105 \%$ of the chord length and enables to measure high-frequency pressure oscillations generated by the bubble clouds shed from the hydrofoil trailing edge. The second variant of the hydrofoil has 5 integrated PVDF films (Figure 5), which are flush with the hydrofoil surface and allow us to quantify the forces produced by the violent collapses of the cavitation structures.
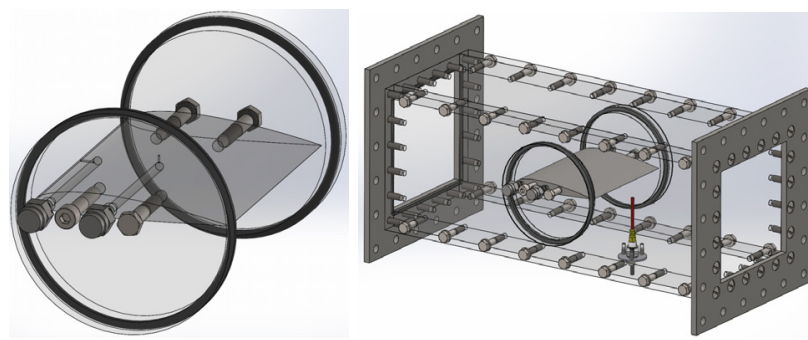

Figure 4. Variant of hydrofoil with static pressure probes.
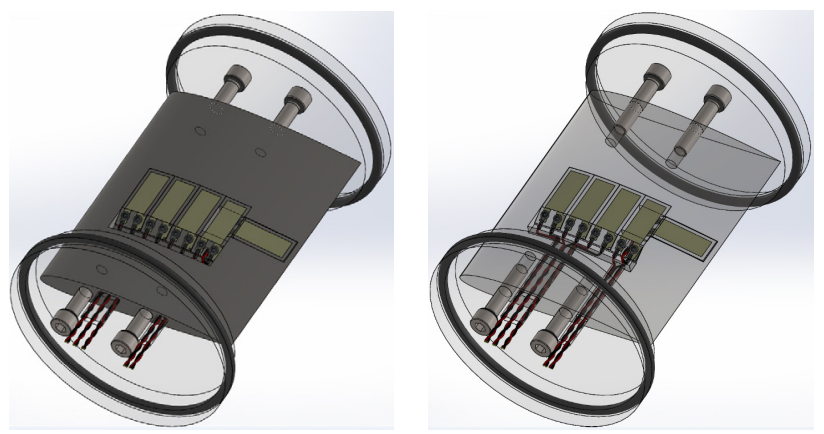

Figure 5. Variant of hydrofoil with PVDF films.

\section{Instrumentation}

The volume flow rate through the test section is measured by the magnetic-inductive flowmeter with the accuracy class $0.3 \%$. The static pressures are measured using the transducers with the accuracy class $0.5 \mathrm{kPa}$. The diameter of the static pressure tap holes is $1 \mathrm{~mm}$ and the natural frequency of the pressure measurement system is about $4.5 \mathrm{kHz}$. Typically, the pressures are measured with the sampling frequency $5 \mathrm{kHz}$, with each record lasting 10 seconds. Special attention has been paid to the installation of the PVDF hydrophone downstream of the hydrofoil. The hydrophone consists of a thin rod attached to its base using a flexible cable (Figure 6). The hydrophone is protected by a conical holster, which eliminates the vibrations of the hydrophone tip at high flow rates. This configuration also helps to seal the hydrophone cable from the moisture. The holster is equipped with a small cap, which protects the active hydrophone tip from the cavitation erosion damage during the extended periods of visualization experiments.

The temperature of the flowing water is measured using the platinum thermometer in the class of accuracy $0.5 \%$ located in the water reservoir. To enable monitoring of the nuclei content in the inlet flow a bypass section is installed allowing to apply the acoustic bubble spectrometer. The visualizations are performed by two high-speed cameras simultaneously recording the top and side views of the hydrofoil. The recording frequency is $1000 \mathrm{fps}$, the image resolution $1024 \times 1024$ pixels and the length of each record is 2 seconds. The static pressure measurements are synchronized with the cameras.
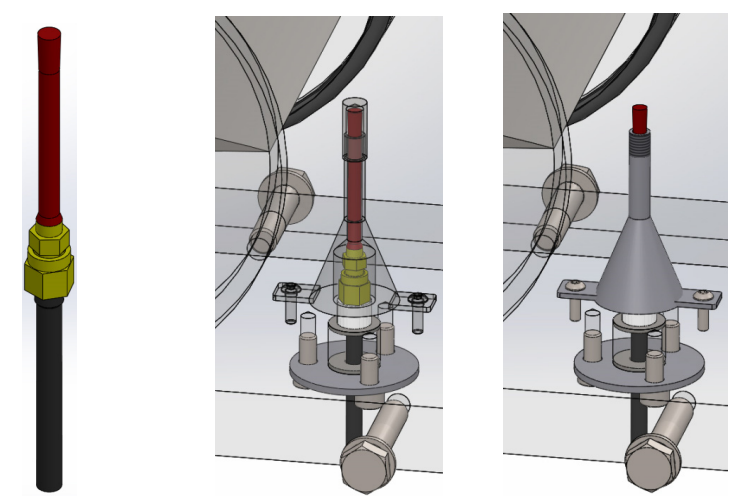

Figure 6. PVDF hydrophone and its holster with cap.

\section{CFD tools and computational model}

A comprehensive CFD analysis has been carried out in order to determine the range of regimes for the experimental study. The ANSYS CFX software has been used to solve the unsteady Reynolds-averaged NavierStokes equations. The interphase mass transfer has been described using the Rayleigh-Plesset model included in the ANSYS CFX package within the homogenous multiphase model. The highly unsteady phenomena have been modelled using the SST turbulence model as well as some advanced turbulence models available in the ANSYS CFX, namely the BSL-based Reynolds-stress model, the SSG Reynolds-stress model and the SAS-SST model. The SAS-SST model showed the best agreement with the measured frequencies of the cavitation-induced flow oscillations and the experimental visualizations. The future work will, however, require more detailed analyses with very fine computational grids and non-URANS turbulence models such as the LES or DES simulations. The boundary conditions are based on the prescribed total pressure at the inlet and the mass flow rate at the outlet. No symmetry plane at the hydrofoil midspan has been used.

Three different incidence angles have been tested numerically $\left(0^{\circ}, 4^{\circ}\right.$ and $\left.8^{\circ}\right)$. The most interesting phenomena have been detected for the incidence 
angle $8^{\circ}$. Therefore, most results have been obtained for this particular incidence angle. Figure 7 shows the Reynolds numbers (Re) and the cavitation numbers $(\sigma)$ used in the CFD analysis and in the experiments for the incidence angle $8^{\circ}$. The Reynolds number is defined as

$$
\operatorname{Re}=\frac{u_{r e f} C}{v}
$$

where $u_{\text {ref }}$ is the velocity at the test section inlet, $C$ is the hydrofoil chord length, and $v$ is the kinematic viscosity of the liquid. The cavitation number is defined as

$$
\sigma=2 \frac{\left(p_{r e f}-p_{v}\right)}{\rho u_{r e f}^{2}}
$$

where $p_{\text {ref }}$ is the reference static pressure at the inlet of the test section, $p_{\mathrm{v}}$ is the vapour pressure, and $\rho$ is the density of the liquid. The minimum cavitation number in the experiments is limited by the operating capacity of the tunnel's main driving pump.

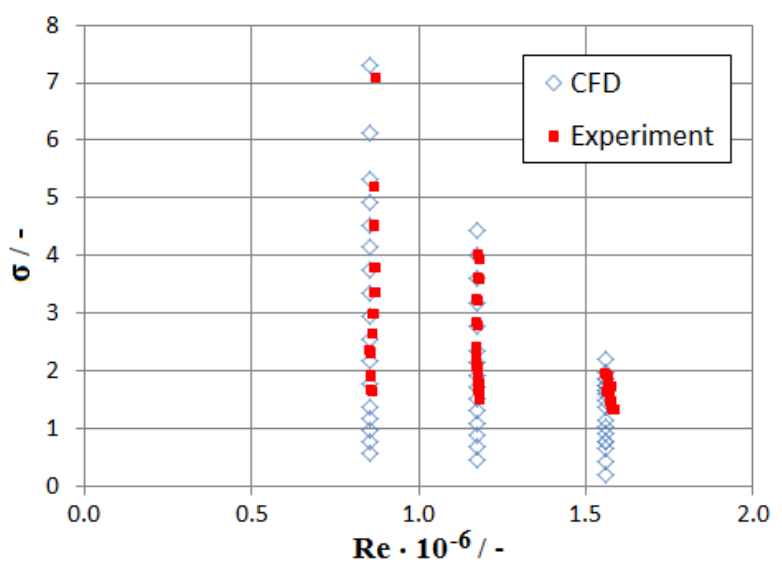

Figure 7. Reynolds numbers Re and cavitation numbers $\sigma$ used in the CFD analysis and in the experiments.

\section{Preliminary measurements and comparison with CFD simulation}

The first experiments for the incidence angle of $8^{\circ}$ and the regime of partial cavitation have shown that the dominant frequency of the pressure oscillations depends strongly on the cavitation number and that for a certain range of cavitation numbers a "resonance" effect can be observed. This resonance effect is manifested by the dramatic increase of the amplitudes of the pressure oscillations on the suction side of the hydrofoil. The FFT analysis of the static pressure P40 measured by the pressure transducer located at $40 \%$ of the hydrofoil chord length for similar Reynolds numbers and different cavitation numbers (Figure 8) indicates that the amplitudes of the pressure oscillations in the "resonant" regime (for $\sigma=1.71$ ) are nearly one order of magnitude higher than for sufficiently high or low cavitation numbers. The camera recordings show that the sheet cavities formed on the hydrofoil are small and relatively stable when $\sigma$ is sufficiently high. There is only one dominant frequency about $21 \mathrm{~Hz}$ (Strouhal number
$\mathrm{St} \approx 0.2, \mathrm{St}=f C / u_{\text {ref }}$, where $f$ is the shedding frequency) and the amplitudes are rather low. As the cavitation number is decreased (i.e. the cavitation intensity is increased) two (or more) significant frequencies can be identified. The lowest frequency (about $15 \mathrm{~Hz}$, which corresponds to $\mathrm{St} \approx 0.14$ ) is the most significant. In this regime, very intensive interaction of the cavitation structures with the separated flow on the suction side of the hydrofoil is observed. The further decrease of $\sigma$ causes a decrease of the amplitudes and for sufficiently low cavitation numbers a stable supercavitation regime is developed, which does not produce any dominant frequencies. In the supercavitation regime the backflow on the suction side of the hydrofoil practically vanishes. In the experiments, the resonant regime is quite narrow (in terms of $\sigma$ ), which makes it difficult to detect. The data from the CFD analysis show the same tendencies, however, the resonant regime is much wider.
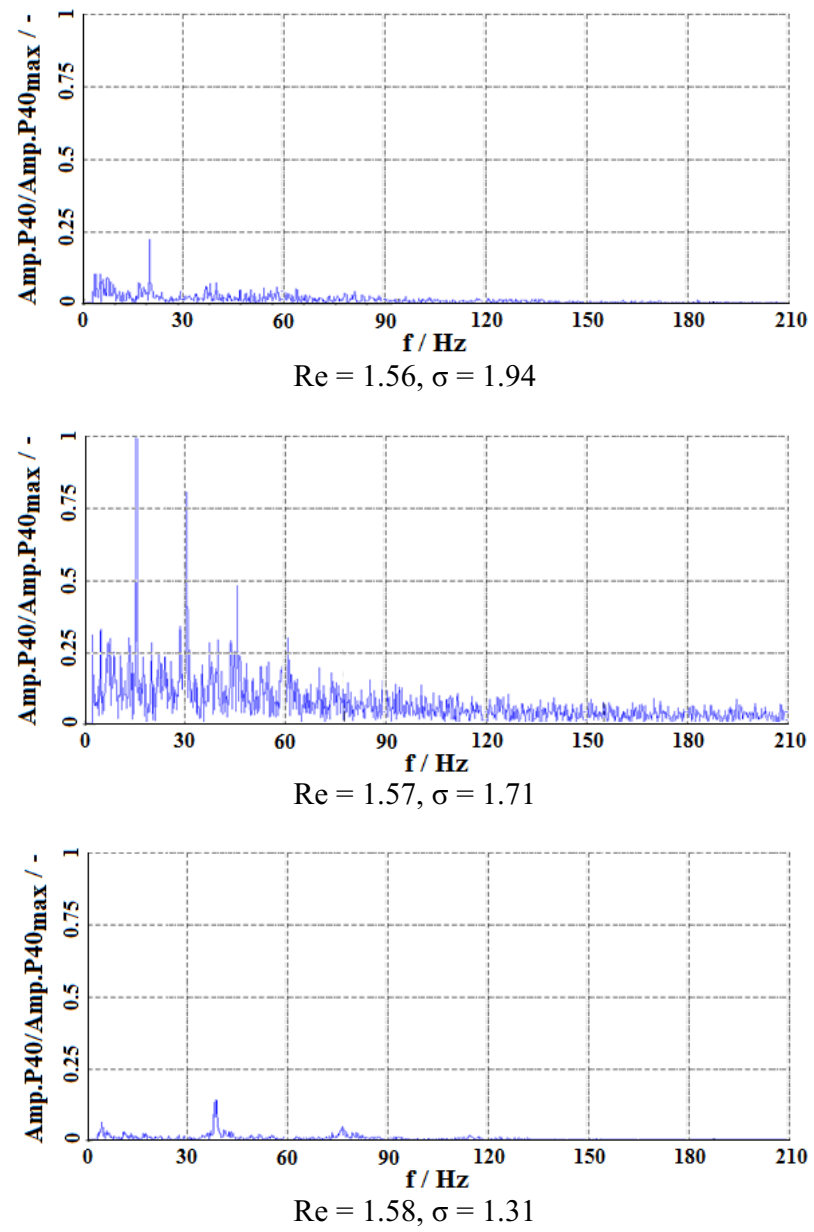

Figure 8. FFT analysis of static pressure $\mathrm{P} 40$ for different cavitation numbers.

An interesting feature of the CFD analysis is the perfect periodicity of the pressure oscillations. The maxima of the pressure $\mathrm{P} 40$ reach about $170 \mathrm{kPa}$. The pressure oscillations obtained from the experiments are much less regular and their maxima change from about $100 \mathrm{kPa}$ to $250 \mathrm{kPa}$ (Figure 9). In addition, the dominant frequencies are not so stable and fluctuate in the range $\pm 3 \%$ around the main value, which on the other hand corresponds very well with the numerical result. 


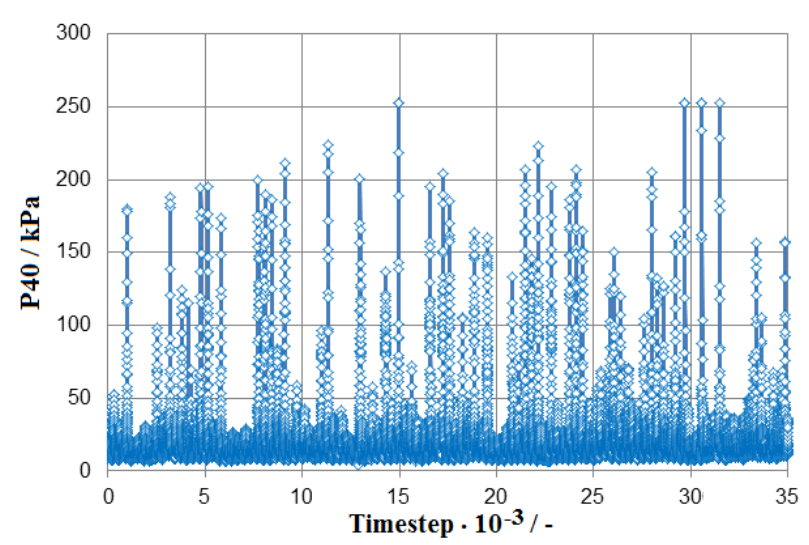

Figure 9. Changes of static pressure $\mathrm{P} 40$ during measurement. $\operatorname{Re}=1.57, \sigma=1.71$
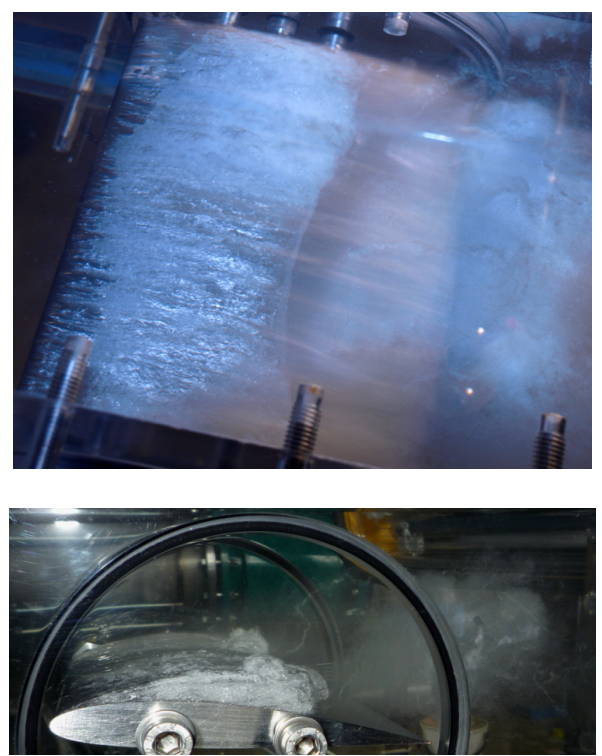

Figure 10. Sheet cavity on hydrofoil before its break-up. Highspeed camera images. $\operatorname{Re}=1.57, \sigma=1.71$.

The preliminary visualizations have provided us with the idea about the periodic interaction between the flow separation and the cavitation on the suction side of the hydrofoil in the resonant regime. First, a sheet cavity is formed behind the leading edge. The cavity grows as it translates towards the trailing edge (Figure 10). Subsequently, a re-entrant jet is formed above the trailing section of the hydrofoil and propagates upstream. When the sheet cavity closure reaches the trailing section of the hydrofoil, it becomes unstable due to the interaction with the re-entrant jet and breaks up. Its main part retracts upstream and a smaller cloud cavity is formed. The cloud cavity is detached from the hydrofoil and moves towards the trailing edge. It then rapidly increases and later vanishes downstream of the hydrofoil, in the area of increased static pressure, to occasionally form a number of worm-like cavitation vortices. Then, a new cycle starts over with a germ sheet cavity formed just behind the leading edge. The coherent collapse of the bubble cloud generates a high impact pressure acting on the hydrofoil surface. Figure 11 shows the creation of a large cloud cavity in the trailing section of the hydrofoil after the break-up of the sheet cavity.
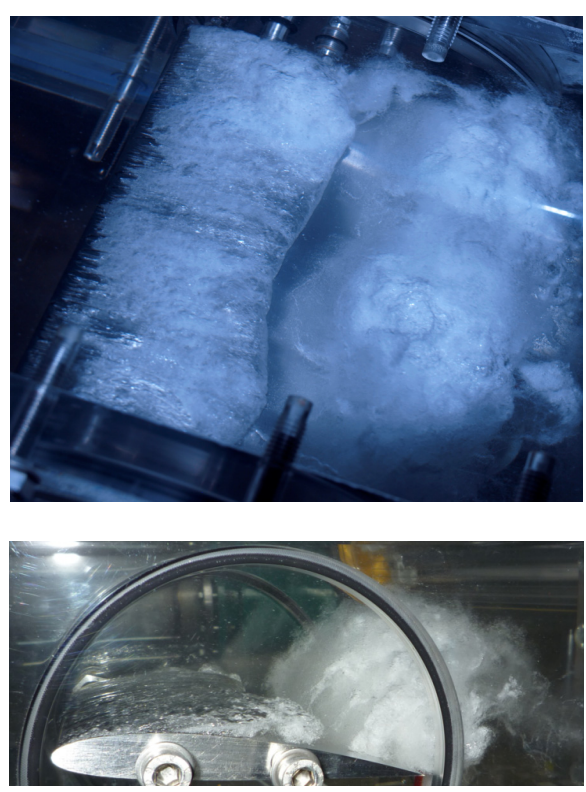

Figure 11. Creation of large cloud cavity in trailing section of hydrofoil. High-speed camera images. $\operatorname{Re}=1.57, \sigma=1.71$.

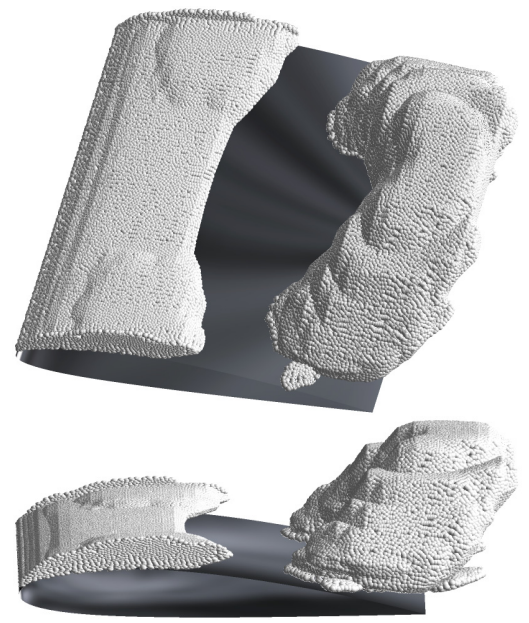

Figure 12. Creation of large cloud cavity in rear part of hydrofoil. $\mathrm{CFD} . \operatorname{Re}=1.57, \sigma=1.71$.

The visualization shown in Figure 11 can be compared with the results of the CFD analysis shown in Figure 12. The white clouds representing the cavitation structures are regions, in which the volume fraction of vapour exceeds $10 \%$. The numerical results provide a detailed insight into the flow field during the periodic processes on the hydrofoil and in its vicinity because 3D data from the CFD simulation can be postprocessed in many illustrative ways. It is possible to apply the graphical tools as well as to evaluate some integral quantities, such as drag, lift or torque. Figure 13 shows the distribution of the volume fraction of vapour in the symmetry plane of the test section. It shows that the cloud cavity separates from the solid surface before it vanishes behind the hydrofoil trailing edge.

As mentioned above, the high-amplitude pressure oscillations in the resonant regime are based on the periodic interaction of the flow separation and the cavitation structures. The experimental investigation of the separated flow close to the hydrofoil would be 
extremely difficult as any potential probes would influence the flow and the cavitation phenomena. That is why the CFD analysis is very useful and complements the experimental data. Figures 14-15 give a good idea about the range and the topology of the flow separation on the suction side of the hydrofoil in the time step captured in Figure 11. In Figure 14, the backflow region (the region where the longitudinal velocity is negative and the fluid flows in the direction from the trailing edge to the leading edge) is visualized. Figure 15 illustrates the surface streamlines on the suction side of the hydrofoil. We can observe the typical U-shaped separation line at the front of the hydrofoil as well as two dominant vortices formed due to the presence of the solid side walls.

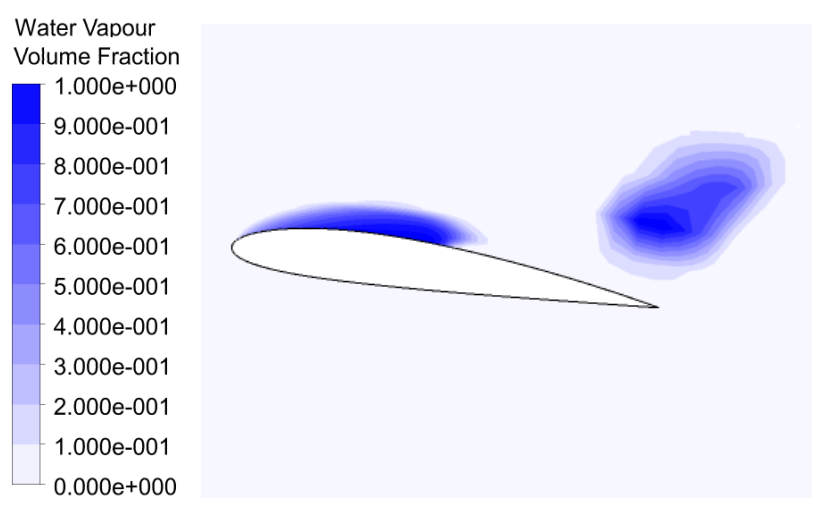

Figure 13. Volume fraction of vapour in the symmetry plane of the test section corresponding to Figure 11.

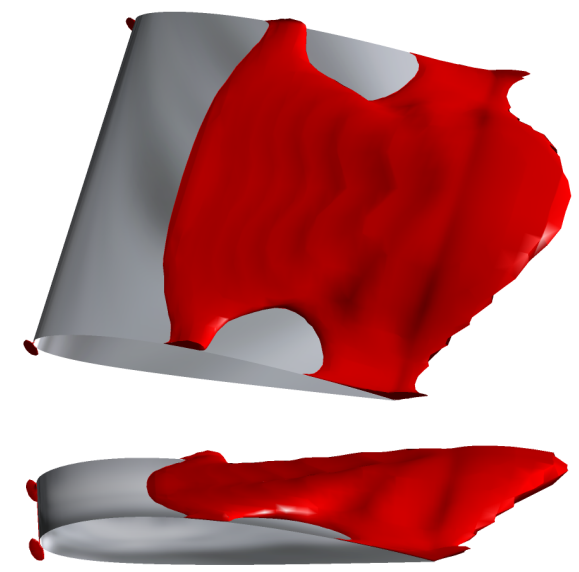

Figure 14. Back-flow flow region on the suction side and behind the trailing edge of hydrofoil (red/dark) corresponding to Figure 11 .

\section{Conclusion}

The aim of this work has been to design an upgraded version of the water cavitation tunnel for the experimental investigation of highly unsteady cavitation phenomena on two-dimensional hydrofoils and to verify its function. The upgraded cavitation tunnel adds the following features to the previous design: (i) ability to measure static pressure on the hydrofoil; (ii) ability to measure impact forces from collapsing bubbles; (iii) ability to operate independently from the hydraulic test rig; (iv) ability to measure pressure oscillations in the far field; (v) unrestricted access to test section for highspeed camera visualization. Still remains the limitation of the minimum cavitation number in the experiments rising from the operating capacity of the tunnel's driving pump.

The preliminary experimental results obtained for the prismatic NACA 2412 hydrofoil have provided a useful and illustrative picture of the cavitation structures on the hydrofoil as well as the corresponding static pressure oscillations for a wide range of inlet flow conditions. The results agree very well with the preliminary CFD analysis. Highly valued is the fact that it has been possible to detect cavitation instabilities (manifested as the resonance effect) both experimentally and by CFD.

The objective for the nearest future is to measure the forces exerted by the collapsing cavitation structures on the hydrofoil surface using the PVDF films and improve the visualization of the cavitation structures using the vertical light sheet located in the symmetry plane of the hydrofoil.

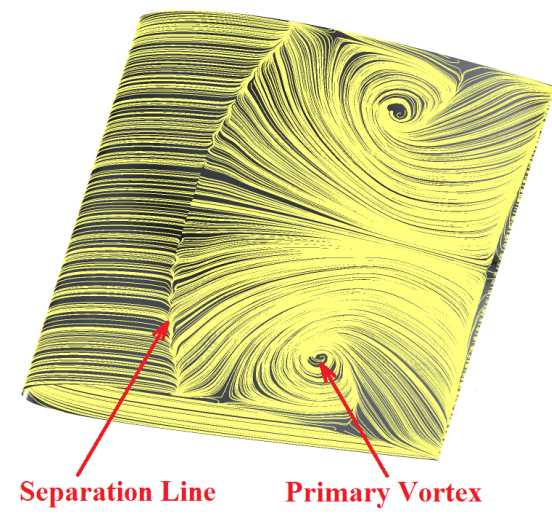

Figure 15. Surface streamlines on the suction side of hydrofoil corresponding to Figure 11.

\section{Acknowledgments}

This work has been supported by the Czech Science Foundation under the grant no. 101/13/23550S "Experimental Research and Mathematical Modelling of Unsteady Phenomena Induced by Hydrodynamic Cavitation".

\section{References}

1. Y. Tsujimoto, Y. Yoshida, M. Maekawa, S. Watanabe, T. Hashimoto, ASME Jour. Fluids Engng., 119, 4 (1997)

2. K. Yamamoto,,Y. Tsujimoto, IJFMS, 2, 1 (2009)

3. Y. Tsujimoto, Fluid Dynamics of Cavitation and Cavitating Turbopumps (Springer-Verlag, 2009)

4. M. Sedlář, M. Bajorek, J. Šoukal, Proc $7^{\text {th }}$ European Conference on Turbomachinery (Athens, 2007)

5. N. Yamanishi, S. Fukao, X. Qiao, C. Kato, Y. Tsujimoto, ASME J. Fluids Engng., 129 (2007)

6. M. Sedlář, O. Šputa, M. Komárek, IJFMS, 5, 1 (2012)

7. M. Sedlár̆, M. Komárek, M. Vyroubal, M. Müller, EPJ Web of Conferences, 25 (2012)

8. S. Watanabe, Y. Konishi, I. Nakamura, A. Furukawa, Proc. WIMRC $3^{\text {rd }}$ Int. Cav. Forum (Warwick, 2011) 